\title{
Sleep Spindles Promote the Restructuring of Memory Representations in Ventromedial Prefrontal Cortex through Enhanced Hippocampal-Cortical Functional Connectivity
}

\author{
Emily Cowan, ${ }^{1}$ Anli Liu, ${ }^{2,3}$ Simon Henin, ${ }^{2,3}$ Sanjeev Kothare, ${ }^{2,3}$ (C) Orrin Devinsky, ${ }^{2,3}$ and $\odot$ Lila Davachi ${ }^{4,5}$ \\ ${ }^{1}$ Center for Neural Science, New York University, New York, New York 10003, ${ }^{2}$ Comprehensive Epilepsy Center, New York University, New York, New York \\ 10016, ${ }^{3}$ Department of Neurology, New York University Langone Health, New York, New York 10017, ${ }^{4}$ Psychology Department, Columbia University, \\ New York, New York 10027, and ${ }^{5}$ Nathan Kline Institute, Orangeburg, New York 10962
}

\begin{abstract}
Memory consolidation is hypothesized to involve the distribution and restructuring of memory representations across hippocampal and cortical regions. Theories suggest that, through extended hippocampal-cortical interactions, cortical ensembles come to represent more integrated, or overlapping, memory traces that prioritize commonalities across related memories. Sleep processes, particularly fast sleep spindles, are thought to support consolidation, but evidence for this relationship has been mostly limited to memory retention benefits. Whether fast spindles provide a mechanism for neural changes hypothesized to support consolidation, including the strengthening of hippocampal-cortical networks and integration across memory representations, remains unclear, as does the specificity of regions involved. Using functional connectivity analyses of human fMRI data (both sexes), we show that fast spindle density during overnight sleep is related to enhanced hippocampal-cortical functional connectivity the next day, when restudying information learned before sleep. Spindle density modulated connectivity in distinct hippocampal-cortical networks depending on the category of the consolidated stimuli. Specifically, spindle density correlated with functional connectivity between anterior hippocampus and ventromedial prefrontal cortex (vmPFC) for object-word pairs, and posterior hippocampus and posteromedial cortex for scene-word pairs. Using multivariate pattern analyses, we also show that fast spindle density during postlearning sleep is associated with greater pattern similarity, or representational overlap, across individual object-word memories in vmPFC the next day. Further, the relationship between fast spindle density and representational overlap in vmPFC was mediated by the degree of anterior hippocampal-vmPFC functional connectivity. Together, these results suggest that fast spindles support the network distribution of memory traces, potentially restructuring memory representations in vmPFC.
\end{abstract}

Key words: fMRI; hippocampus; memory consolidation; sleep; sleep spindles; vmPFC

Significance Statement

How new experiences are transformed into long-term memories remains a fundamental question for neuroscience research. Theories suggest that memories are stabilized as they are reorganized in the brain, a process thought to be supported by sleep oscillations, particularly sleep spindles. Although sleep spindles have been associated with benefits in memory retention, it is not well understood how spindles modify neural memory traces. This study found that spindles during overnight sleep correlate with changes in neural memory traces, including enhanced functional connectivity in distinct hippocampal-cortical networks and increased pattern similarity among memories in the cortex. The results provide critical evidence that spindles during overnight sleep may act as a physiological mechanism for the restructuring of neural memory traces.

\section{Introduction}

Accumulating evidence suggests sleep plays an important role in the retention of memories (Diekelmann and Born, 2010; Rasch

This work was supported by National Institutes Health Grant R01-MH-076492 to L.D., a Dart Neuroscience Grant to L.D., a NYU Clinical Translational Science Initiative Grant to A.L., and a National Science Foundation and Born, 2013). However, precisely how sleep alters neural memory traces to support memory is still not well understood. Consolidation theories posit that new memories become stabi- 
lized as they are gradually reorganized and distributed across neocortical networks (Alvarez and Squire, 1994; McClelland et al., 1995). This memory transformation process is thought to be supported, at least partly, by neural oscillations occurring during non-rapid eye movement (nonREM) sleep (Sirota et al., 2003; Diekelmann and Born, 2010; Rasch and Born, 2013). For example, prior research has consistently linked sleep spindles (11-16 $\mathrm{Hz}$ ) with benefits for later memory performance (Gais et al., 2002; Clemens et al., 2005, 2006; Mednick et al., 2013; Rasch and Born, 2013). Sleep spindles are proposed to help coordinate neural activity between the hippocampus and neocortex through interactions with sharp-wave ripples $(80-100 \mathrm{~Hz})$, oscillations supporting hippocampal replay (Wilson and McNaughton, 1994; Siapas and Wilson, 1998; Clemens et al., 2011), and cortical slow oscillations ( $\sim 0.5-1$ Hz; Mölle et al., 2002; Clemens et al., 2007; Staresina et al., 2015; Klinzing et al., 2016). Indeed, during sleep, fast spindle activity is coincident with increases in univariate activation in hippocampus and cortex (Schabus et al., 2007; Bergmann et al., 2012) and hippocampal-cortical functional connectivity (Andrade et al., 2011), as well as the reactivation of memories (Schönauer et al., 2017; Antony et al., 2018; Cairney et al., 2018).

While these data broadly support the notion that spindle activity promotes memory consolidation, gaps remain in our current understanding about this relationship. If spindles act as a mechanism for systems consolidation, they should be related to neural markers of consolidation that endure after sleep-yet, little work has examined how spindles affect neural memory representations. Here, we address three critical questions concerning the way sleep spindles effect lasting changes on the neural organization of memories.

First, a central tenant of systems consolidation models is the enhanced communication between hippocampus and cortex. Prior work has shown that hippocampal-cortical functional connectivity increases with time (Gais et al., 2007; Sterpenich et al., 2007; Vilberg and Davachi, 2013; but see: Takashima et al., 2009; Baran et al., 2016), in a manner predicting memory retention (Vilberg and Davachi, 2013). However, whether spindles during sleep contribute to such enhancements in hippocampal-cortical functional connectivity remains unknown. We additionally queried the specificity of these effects. The anterior and posterior hippocampus have been shown to participate in distinct networks with differing functional specialization, processing object/ item information or spatial relationships/context, respectively (Davachi, 2006; Libby et al., 2012; Ranganath and Ritchey, 2012; Nadel et al., 2013; Poppenk et al., 2013; Robin and Moscovitch, 2017). Using two stimulus categories, we tested whether the relationship between spindles and hippocampal network connectivity is sensitive to the content of the presleep information.

Second, models of consolidation suggest that the cortex gradually extracts the central features ("gist") from new memories (Alvarez and Squire, 1994; McClelland et al., 1995; Robin and Moscovitch, 2017), resulting in more integrated traces. Prior work has shown that mPFC representations for similar experiences become more integrated, or overlapping, with time (Richards et al., 2014; Tompary and Davachi, 2017). Yet, it remains unclear whether spindles during sleep contribute to this neural restructuring process.
Finally, connecting the preceding hypotheses, we tested whether the reorganization of cortical memory representations depends on the modulation of hippocampal-cortical networks. If sleep spindles promote the transfer of information through hippocampal-cortical interactions, we hypothesized the magnitude of neural integration across cortical traces would be related to the strength of hippocampal-cortical functional connectivity.

We designed a $3 \mathrm{~d}$ within-participant experiment that included presleep and postsleep encoding, measures of overnight polysomnography (PSG), a next-day fMRI scan, and behavioral memory tests (Fig. 1). We examined how spindles measured during the intervening night relate to differences in functional connectivity and multivariate pattern similarity for memories learned before, versus after, sleep.

\section{Materials and Methods}

Participants. A total of 22 participants ( 7 female, 15 male) were recruited for this experiment (all protocols approved by the New York University School of Medicine Institutional Review Board). Two participants were excluded due to problems with the MRI scanner, and one participant was excluded as an outlier for total sleep time $(>2.5$ SDs below the group average). All participants were between 18 and 35 years of age (mean age $=25.3$ years); fluent in English; did not have any diagnoses of neurologic, psychiatric, or sleep disorders; and were not using any psychoactive medications. Participants were included if they had not traveled across time zones or completed night-shift work in the month preceding the first study session, had a body mass index of $<30$, and did not have any contraindications for the MRI. For the $48 \mathrm{~h}$ before the beginning of session 1 (day 1), participants were asked to log their sleep patterns, refrain from any alcohol or drug use, and reduce caffeine intake to one cup per day. At the beginning of session 1, participants were asked to complete questionnaires to assess eligibility for study participation and to query sleep habits, including the MOCA, Insomnia Symptom Questionnaire, Morning-Eveningness Questionnaire, STOP-BANG Questionnaire, and Epworth Sleepiness Scale. All participants provided informed consent and were compensated for their time. The sample size was based on similar studies examining sleep effects on univariate neural activation (Takashima et al., 2006; Gais et al., 2007; Sterpenich et al., 2007; Hennies et al., 2016).

Experiment design. Participants completed a $3 \mathrm{~d}$ experiment, as outlined in Figure 1. Each participant studied three separate Encoding Lists, consisting of unique scene-word and object-word pairs. The Sleep List (SL) was encoded before the overnight sleep period (day 1), during which polysomnography was recorded (lights out at $\sim 10: 30$ P.M.), while the Morning List (ML) was encoded after awakening the next morning (at $\sim 7: 30$ A.M., day 2). Participants were provided $\sim 30 \mathrm{~min}$ to eat breakfast before beginning the Morning List encoding session. Approximately $2 \mathrm{~h}$ later, both the Sleep List and Morning List were studied for a second time in the fMRI scanner, intermixed with a third, novel list of word-image 
pairs (Single Study List, SS). Participants completed two source memory tests, immediately after the scan (day 2) and after a 24 h delay (day 3 ).

Encoding. For the encoding sessions, each trial consisted of the presentation of a central red fixation cross for $500 \mathrm{~ms}$, followed by a word and image (scene or object) pair for $4500 \mathrm{~ms}$, then a black fixation cross for $500 \mathrm{~ms}$. Participants were instructed to form a vivid mental association between the word and image on screen, and then rate how well the association could be formed, responding either "very well," "somewhat well," or "not well." Each of the three Encoding Lists consisted of 120 word-image pairs ( 60 object-word and 60 scene-word pairs). Encoding sessions were divided into two blocks of 60 trials, separated by a $30 \mathrm{~s}$ break. The order of word-image pairs was randomized for each participant and intermixed between the categories. Word-image pairs were counterbalanced across Encoding Lists and across participants. Between trials, participants completed an active baseline task for $8.5 \mathrm{~s}$ (Stark and Squire, 2001). Numeric digits ranging between 1 and 9 were presented, and participants were instructed to respond "even" or "odd." Digits were presented for $2 \mathrm{~s}$ each or until a response was entered, and followed by a black fixation cross for $250 \mathrm{~ms}$. Outdoor scene stimuli were randomly selected from an online database (http://olivalab.mit.edu/MM/; Oliva and Torralba, 2001). Object stimuli were selected from the MIT Massive Memory set (Brady et al., 2008), and word stimuli were adjectives from the MRC (Medical Research Council) psycholinguistics database (http:// websites.psychology.uwa.edu.au/school/MRCDatabase/uwa_mrc.htm). Words were presented in size 36 black Helvetica font on a white background. All stimuli were presented on an Apple MacBook laptop, and responses were made using the computer keyboard.

Overnight polysomnography. Overnight polysomnography (PSG) was conducted at the NYU Langone Sleep Center using the Xltek Data Acquisition System (Natus Medical). PSG measurements included standard electroencephalography (International 10/20 electrode placement; Fp1, Fp2, F7, F3, F8, F4, Fz, Cz, T3, C3, C4, T4, T5, P3, Pz, P4, T6, O1, O , and A1, A2 mastoid references), electrooculogram (EOG; left/right), chin electromyogram, as well as chest and leg movements, respiratory monitoring, and blood oxygenation. EEG data were digitized at a sampling rate of $256 \mathrm{~Hz}$ and filtered between 0.1 and $70 \mathrm{~Hz}$.

fMRI session. Participants traveled to the Center for Brain Imaging at New York University for the fMRI session. In the scanner, participants were re-presented with all previously studied word-image pairs from both the Sleep and Morning Lists, as well as novel pairs ("Single Study List"); during the scanning session, the 120 word-image pairs from each list were randomly intermixed, and divided into six separate runs, with 60 trials per run. Participants performed the same task as in the previous two encoding sessions (forming an association between the word and image, rate how well they could do so). Trials were presented with the same stimulus timing as described above for the behavioral encoding sessions and again included an odd/even active baseline task between trials. All responses were made using an MRI-compatible button box. After the encoding task, participants completed a localizer task in which they viewed novel objects, scenes, and scrambled objects, which was used in a separate line of inquiry. A high-resolution anatomical image of each participant's brain was acquired at the end of the scan.

fMRI parameters and preprocessing. Scanning was completed on a $3 \mathrm{~T}$ Siemens Allegra head-only scanner. Functional imaging data were collected using an echoplanar imaging pulse sequence $(\mathrm{TR}=2000 \mathrm{~ms}, \mathrm{TE}=$ $15 \mathrm{~ms}, 34$ interleaved slices oriented parallel to the anterior commissureposterior commissure axis, flip angle $=82^{\circ}$, voxel size $=3 \times 3 \times 3 \mathrm{~mm}$ ). The first six volumes were discarded to allow for T1 stabilization. A high-resolution T1-weighted anatomical scan (magnetization-prepared rapid-acquisition gradient echo sequence, voxel size $=1 \times 1 \times 1 \mathrm{~mm}$ ) was acquired to aid in functional image coregistration.

Preprocessing on functional data were performed using the FSL (version 5.0.2.2) fMRI Expert Analysis Tool version 6 (FSL: http://fsl.fmrib. ox.ac.uk/fsl/). Functional images were brain extracted, high pass filtered (110 s cutoff), and realigned to correct for interleaved acquisition, and MCFLIRT was applied for motion correction. For psychophysiological interaction analyses, data were spatially smoothed with a $5 \mathrm{~mm}$ FWHM kernel. The FSL motion outliers tool was used to identify outlier time points, which were included as an additional regressor in subsequent general linear models (GLMs) to remove the outlier time points. Functional data were registered to the high-resolution anatomical scans with the FSL FLIRT tool (12 df; nonlinear registration, $10 \mathrm{~mm}$ warp resolution), then to standard Montreal Neurological Institute space using FNIRT nonlinear registration with a $10 \mathrm{~mm}$ warp resolution.

Region of interest definition. Anatomical participant-specific hippocampal regions of interest (ROIs) were defined using the FSL automatic segmentation tool FIRST. For each participant, left and right hippocampal masks were generated, and, in MATLAB, were divided lengthwise into anterior and posterior halves.

Since the anatomical boundaries of the vmPFC are not well defined, we adopted a vmPFC ROI previously identified in an article examining changes in univariate activation over time, including a period of sleep (Takashima et al., 2006; coordinates: $-2,32,-10$ ). In line with this prior study, these coordinates were used to create an $8 \mathrm{~mm}$ kernel sphere. Likewise, to define an ROI for posterior medial cortex (PMC), we used a mask available from an online database (findlab.stanford.edu/functional_ ROIs.html; Shirer et al., 2012). Additional cortical ROIs in perirhinal cortex $(\mathrm{PrC})$ and posterior parahippocampal cortex $(\mathrm{PhC})$, which have both been implicated in memory and consolidation-related processes (Davachi, 2006; Ranganath and Ritchey, 2012; Vilberg and Davachi, 2013), were defined from a contrast of average brain activity for all word-image pairs versus baseline, a contrast agnostic to condition or category. These ROIs were generated using an $8 \mathrm{~mm}$ kernel sphere centered on the coordinate of peak activation identified within the known anatomical borders of these regions.

Immediate and delayed memory tests. Immediately after participants were removed from the scanner, their memory for the word-image associations was probed by testing source memory, using the word as a cue. Participants also returned to the laboratory $24 \mathrm{~h}$ later for a second, "delayed" memory test. Participants were asked to try to remember whether they had seen the word before and whether it had been paired with a scene or object image. The possible responses included the following: "old-scene," "old-object," "word only," or "new." The legend for these response options was presented under the cue-word on screen. Participants were instructed to use the "scene" and "object" responses only if they could remember the specific image with which the word had been paired, while the "word-only" key was to be used if they recognized the test word but could not remember the associated category of the image it had been paired with. The "new" key was to be used if participants believed the word to be novel, or if they were unsure whether it had been studied. Half of the words from each Encoding List (30 object-word, 30 scene-word) were included on each of the two tests (immediate and delayed) to avoid retesting stimuli, along with 60 novel foil words. Each test trial consisted of a red fixation cross presented for $500 \mathrm{~ms}$, followed by a centrally presented word cue, which was on screen for a maximum of $12 \mathrm{~s}$, or until a response was made, and was followed by a black fixation cross for $200 \mathrm{~ms}$.

Statistical analyses. All reported statistical analyses are two tailed. Repeated-measures ANOVAs were performed and followed up with paired sample $t$ tests where applicable. Statistical tests were used to test the difference between correlations, including between two dependent correlations sharing a variable (Williams's test; e.g., functional connectivity correlations with fast spindle density) or between two dependent correlations with different variables (Steiger test; e.g., connectivity-similarity correlations for remembered vs forgotten pairs) with the R package r.test. Tests of Bayes factor (BF) were computed on relevant analyses using the R package BayesFactor. Statistics were performed with R version 3.5.1, RStudio (version 0.99.903, RStudio) and MATLAB (MathWorks) using both built-in and custom functions. $p<0.05$ was considered significant for all statistical tests. Details for analyses are included in the subsections below.

Behavioral analyses. Correct associative memory was defined as accurate source memory judgments ("remembered"), while misses, those previously studied pairs called new, were counted as "forgotten." For all imaging analyses, memory was collapsed across the immediate and delayed tests to increase statistical power. A $3 \times 2 \times 2$ repeated-measures ANOVA was used to assess for differences in the Encoding List (SL, ML, SS), in Memory Test (immediate, delayed), and in category (object, scene). 
Polysomnography. Each participant's night of sleep was scored by a sleep technician and checked over by a board-certified sleep physician, according to the American Academy of Sleep Medicine manual (Berry et al., 2012). The data were staged in $30 \mathrm{~s}$ epochs and categorized as stage 1 , stage 2, slow-wave sleep (SWS), or REM sleep. Together, the first three stages were defined as non-REM sleep.

To estimate spindle density across a night of sleep, a custom algorithm was implemented in MATLAB (MathWorks). All data were first rereferenced to the left mastoid. Identification of spindles was computed on data from the $\mathrm{Cz}$ electrode from epochs identified as either stage 2 or SWS. Evidence suggests there are two types of spindles: slow $(<12 \mathrm{~Hz})$ and fast $(>12 \mathrm{~Hz})$, differing both in their frequency range and cortical topography (Mölle et al., 2011; Rasch and Born, 2013). As fast spindles have more consistently been associated with hippocampal processing and memory (Schabus et al., 2007; Andrade et al., 2011; Mölle and Born, 2011; Bergmann et al., 2012; Rasch and Born, 2013; Staresina et al., 2015; Antony et al., 2018), analyses focused on fast spindles (12-16 Hz). To identify fast spindles, a Morlet wavelet filter centered on $14 \mathrm{~Hz}$ was applied to the data (a method similar to that in the study by Wamsley et al., 2012). Spindles were identified if the wavelet power exceeded twice the averaged power over all stage $2 /$ SWS epochs and its duration was between 0.4 and $3 \mathrm{~s}$. For each participant, spindle density was calculated as the average number of spindles per $30 \mathrm{~s}$ epoch.

Functional connectivity analyses. We conducted psychological-physiological interaction (PPI) analyses, measuring how the correlation between activation in a "seed" brain region and activation in other regions is modulated by a psychological parameter. In keeping with our interest in the dynamics of the long axis of the hippocampus, both anatomically defined anterior and posterior hippocampal ROIs were used as seed regions.

Two different types of PPI analyses were performed, as follows: the first assessed functional connectivity for all trials on the Encoding Lists, and the second was broken down by subsequent memory status (remembered vs forgotten). For the all-trial PPI GLMs, task regressors were created for each Encoding List and image category (object or scene), for a total of six regressors (e.g., SL-object, SL-scene, ML-object, ML-scene etc.). The averaged hippocampal time course of activity was extracted from each participant's preprocessed functional data and modeled as a physiological regressor. Finally, four psychological regressors were modeled as the interaction between the hippocampal time course of activity and the Sleep List and Morning List regressors for each category. Two additional contrasts were defined collapsing by category (object + scene analysis).

For the subsequent memory PPI GLMs, 24 regressors were included to model the four possible memory responses: remembered (source correct), source incorrect, word only, or forgotten (miss), broken down by the Encoding List and the category of paired image, collapsed across memory test. As we were interested in comparing functional connectivity for trials with successful associative memory to those later forgotten trials, we specified our analysis to contrasts of remembered and forgotten pairs. Four psychological regressors were modeled as the interaction between the hippocampal time course of activity and the remembered or forgotten pairs from the Sleep List and Morning List, respectively. Separate analyses were conducted for trials with each category of image (object or scene). Contrasts for each condition greater than baseline were then used in second-level analyses (across runs, within participant), modeled using a fixed-effects analysis. From the relevant contrasts, average $\beta$ estimates were extracted from our ROIs to examine task-dependent changes in functional connectivity and correlations with overnight fast spindle density. Paired $t$ tests were used to assess the differences between the Sleep List and Morning List functional connectivity values.

Inter-item pattern similarity analyses. For inter-item pattern similarity analyses, functional data were preprocessed as outlined above with a few exceptions, as follows: the data were smoothed using a $3 \mathrm{~mm}$ FWHM kernel, and were kept in native space for each participant. After preprocessing, each run was aligned to the first run. For each run, trial-level GLMs were constructed by modeling one regressor per trial 60 regressors in total), with additional regressors for extreme head motion and temporal derivative (Mumford et al., 2014; Tompary and Davachi,
2017). Resulting $t$ statistic maps were imported into MATLAB, where $t$ statistic activation maps were extracted for each ROI in native space.

A linear vector containing the BOLD activation in each voxel in each ROI was extracted for each trial in the three Encoding Lists (Sleep List, Morning List, Single Study List), and the Pearson's correlation coefficient $(r)$ was computed between each pairwise vector. This allowed assessment of the extent to which the activation patterns for each trial correlated with all other trials from the same list across scanning runs, thereby avoiding confounds from temporal autocorrelation (Mumford et al., 2014). The averaged trial-level correlations were then normalized using a Fisher $r$-to- $z$ transformation and averaged, resulting in similarity estimates for each Encoding List (see Fig. 4a). Correlations were performed in the following two ways: first, with all other trials from the same Encoding List, regardless of later memory outcome, and separately only among later remembered or forgotten trials from the same Encoding List ("subsequent memory"). Akin to earlier studies, this analysis allowed for the examination of representations among memories learned at the same time (LaRocque et al., 2013; Tompary and Davachi, 2017; Ezzyat et al., 2018): $3 \times 2$ repeated-measures ANOVAs were used to assess for differences on the Encoding List (SL, ML, SS) and in category (object, scene).

Mediation analysis. To test the hypothesis that the extent of hippocampal-neocortical functional connectivity mediates the relationship between fast spindles and representational overlap in vmPFC, we used a mediation analysis. Formally, this model tested whether the influence of the measured fast spindle density during overnight sleep on the interitem pattern similarity in vmPFC can be accounted for by the mediator variable, the functional connectivity between anterior hippocampus and vmPFC. For both brain measures, we calculated the sleep-specific metric for the object-word pairs that were later remembered. To test if this effect was significant, we used the bootstrapping method (Shrout and Bolger, 2002), with 10,000 iterations to yield an ACME value (average causal mediation effect), using the mediation package in $\mathrm{R}$.

\section{Results}

\section{Behavioral results}

We expected the twice-presented pairs from the Sleep List and Morning List would be better remembered compared with the once-presented Single Study List pairs. On both the immediate and delayed memory tests, associative memory performance was significantly better for pairs from the Sleep List and Morning List compared with the Single Study List, but memory did not significantly differ between the Sleep List and Morning List (Encoding List main effect: $F_{(2,36)}=57.44, p<0.0001$; Fig. 2). For the Morning List and Single Study List, scene-word pairs were better remembered than object-word pairs on both tests, but for the Sleep List, object-word and scene-word source memories were equated on the immediate memory test (Fig. 2).

\section{Polysomnography: sleep stage duration}

On average, participants slept for a total of $463.13 \mathrm{~min}(\mathrm{SEM}=$ $7.30)$, spending, on average, $23.66 \mathrm{~min}$ in stage $1(\mathrm{SEM}=2.46)$, $233.5 \mathrm{~min}$ in stage $2(\mathrm{SEM}=7.78), 97.74 \mathrm{~min}(\mathrm{SEM}=5.97)$ in SWS, and $108.24 \mathrm{~min}(\mathrm{SEM}=3.65)$ in REM sleep. Our main hypotheses focused on fast spindle density, as fast spindles (12-16 $\mathrm{Hz}$ ) have more consistently been associated with hippocampal processing and memory (Mölle et al., 2011; Rasch and Born, 2013; Staresina et al., 2015; Antony et al., 2018). Fast spindle density was defined for each participant as the average number of spindles identified by a custom algorithm per $30 \mathrm{~s}$ epoch of stage 2 and SWS (see Materials and Methods). The average fast spindle density was $2.1(\mathrm{SEM}=0.14)$.

\section{Functional connectivity}

We hypothesized that, if spindles facilitate hippocampal-cortical interactions in the service of memory consolidation, then spindle density during sleep would correlate with increased functional 


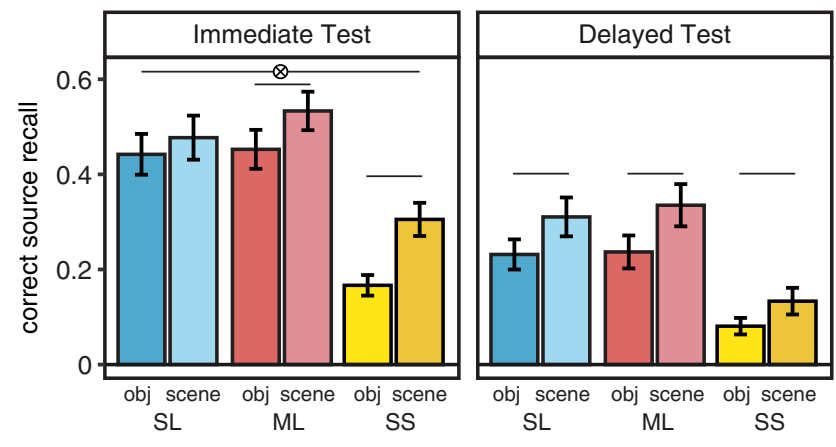

Figure 2. Correct associative memory on immediate and delayed memory tests. Correct source memory was greater for the twice-studied Sleep and Morning List pairs than the once presented Single Study List pairs (main effect of list: $F_{(2,36)}=57.44, p<0.0001 ; S L<S S: p<$ $0.0001 ; \mathrm{ML}<\mathrm{SS}: p<0.0001$ ), and better for the immediate test than delayed test (main effect test: $\left.F_{(1,18)}=136, p<0.001\right)$, consistent with forgetting over time. There was additionally a main effect of category $\left(F_{(1,18)}=13.03, p=0.002\right)$, with scene-word pairs generally better remembered than object-word pairs. A significant list $\times$ category $\times$ test interaction $\left(F_{(2,36)}=\right.$ $4.42, p=0.019)$ seemed driven by a significant difference between accuracy on the object and scene pairs for all comparisons except the Sleep List during the immediate test ( $p=0.21$ ), including the delayed test ( $p=0.036$, object $<$ scene). Lines represent significant differences $p<0.05$, error bars denote SEM.

connectivity measured the next day. We asked whether fast sleep spindles modulate distinct hippocampal-cortical networks based on the content of the restudied pairs (e.g., object-word or sceneword). If fast spindles preferentially impact the networks in a manner that maintains content specificity, we expect a dissociation to emerge when examining functional connectivity in the anterior versus posterior hippocampal networks for the objectword and scene-word pairs, respectively (Davachi, 2006; Hoscheidt et al., 2010; Libby et al., 2012; Ranganath and Ritchey, 2012; Nadel et al., 2013; Robin and Moscovitch, 2017). In particular, anterior hippocampus has greater connectivity with anterior medial temporal lobe regions including perirhinal cortex and the vmPFC, while connectivity is greater between posterior hippocampus and posterior parahippocampal cortex and PMC (Libby et al., 2012; Ranganath and Ritchey, 2012; Eichenbaum, 2017). Thus, we examined functional connectivity between anterior and posterior hippocampus and neocortical regions separately for the object-word and scene-word pairs (see Materials and Methods).

\section{Overnight fast spindle density and next-day functional} connectivity correlations: anterior hippocampus

First, we examined the relationship between overnight fast spindles and next-day anterior hippocampal functional connectivity with targeted neocortical regions during the restudy of paired associate trials from the Sleep List and Morning List (see Materials and Methods). We examined right and left hippocampal seed regions separately, since a 2 (Encoding List) $\times 2$ (hemisphere seed) repeated-measures ANOVA for anterior hippocampalvmPFC functional connectivity resulted in a significant main effect of hemisphere $\left(F_{(1,18)}=6.51, p=0.02\right)$ such that functional connectivity was greater with right, compared with left, anterior hippocampus.

Overnight fast spindle density positively correlated with functional connectivity between right anterior hippocampus and vmPFC measured during the restudy of the Sleep List objectword pairs $(r=0.68, p=0.002, \mathrm{BF}=23.65$; Fig. $3 a)$. Thus, in line with our hypothesis, the greater the density of fast spindles during overnight sleep, the greater the functional connectivity between the anterior hippocampus and vmPFC when restudying the ob- ject-word pairs learned before sleep. Importantly, this relationship was not seen when restudying the more recently learned object-word pairs from the control Morning List $(r=-0.24, p=$ $0.32, \mathrm{BF}=0.71$; Fig. $3 a$ ), which did not have an opportunity to undergo sleep-dependent consolidation. The spindle-connectivity correlations for the two lists were significantly different from each other $(t=2.94, p=0.0097)$, highlighting that the relationship between fast spindle density and Sleep List connectivity does not simply reflect trait-level differences across individuals. Without considering sleep measures, the Sleep List and Morning List functional connectivity values did not significantly differ according to a repeated-measures $t$ test $\left(t_{(18)}=0.07, p=\right.$ $0.94)$, suggesting that overnight spacing versus same-day spacing did not strongly modulate overall connectivity between anterior hippocampus and vmPFC.

To further isolate the relationship between overnight fast spindle density and subsequent functional connectivity, and to normalize for individual differences, we calculated a "sleepspecific" functional connectivity measure by using the Morning List as a direct within-participant control, subtracting each participant's functional connectivity on the Morning List trials from the functional connectivity from the Sleep List trials. The Morning List provided a baseline that could better isolate sleep-specific effects evident for the Sleep List since all other experimental variables (e.g., repeated study, overall performance, average functional connectivity, percept category) are matched for the Sleep List and Morning List. Critically, we see a significant correlation between fast spindle density and this sleep-specific right anterior hippocampal-vmPFC functional connectivity $(r=0.60, p=$ 0.007 ; Fig. $3 b)$. Therefore, even when taking into account the Morning List functional connectivity as a baseline, the density of overnight fast spindles is associated with enhanced network dynamics the next day, when restudying the pairs learned before sleep.

To address the content specificity, we ran the same analyses for the scene-word pairs. Fast spindle density did not significantly correlate with next-day functional connectivity between right anterior hippocampus and vmPFC for either the Sleep List or the Morning List (SL: $r=0.19, p=0.45, \mathrm{BF}=0.61$; ML: $r=$ $0.26, p=0.28, \mathrm{BF}=0.63$ ). However, within the Sleep List, the spindle-connectivity correlations for the object-word and scene-word pairs were only marginally different $(t=1.79, p=0.09)$. There were also no significant correlations with fast spindle density when considering left anterior hippocampal functional connectivity with vmPFC for either object-word or scene-word pairs (object-word pairs: SL $r=0.09, p=0.72$; ML $r=-0.13, p=$ 0.59 ; scene-word pairs: SL $r=0.04, p=0.89$, ML $r=0.03, p=$ $0.9)$, perhaps consistent with the hemispheric difference reported above. Likewise, fast spindle density did not significantly correlate with functional connectivity between right anterior hippocampus and PrC or PMC for either the Sleep List or Morning List pairs of either category, though it did correlate with anterior hippocampal-right $\mathrm{PhC}$ functional connectivity (scene-word pairs: SL $r=0.46, p=0.05$; ML $r=-0.5, p=0.03$ ).

\section{Overnight fast spindle density and next-day functional}

connectivity correlations: posterior hippocampus

By contrast, when analyzing the network involving posterior hippocampus, a different pattern emerged, with the fast spindleconnectivity correlation significant only for the scene-word pairs, and not the object-word pairs. Specifically, right posterior hippocampal-PMC functional connectivity during restudy of the scene-word pairs from the Sleep List positively correlated with 
a

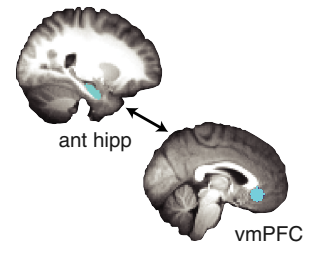

C

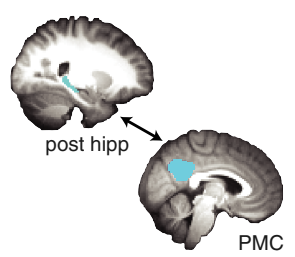

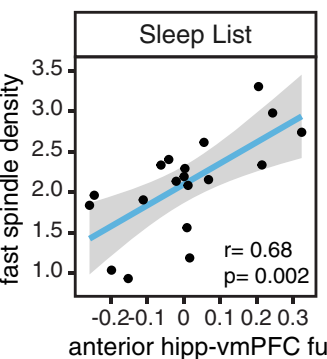
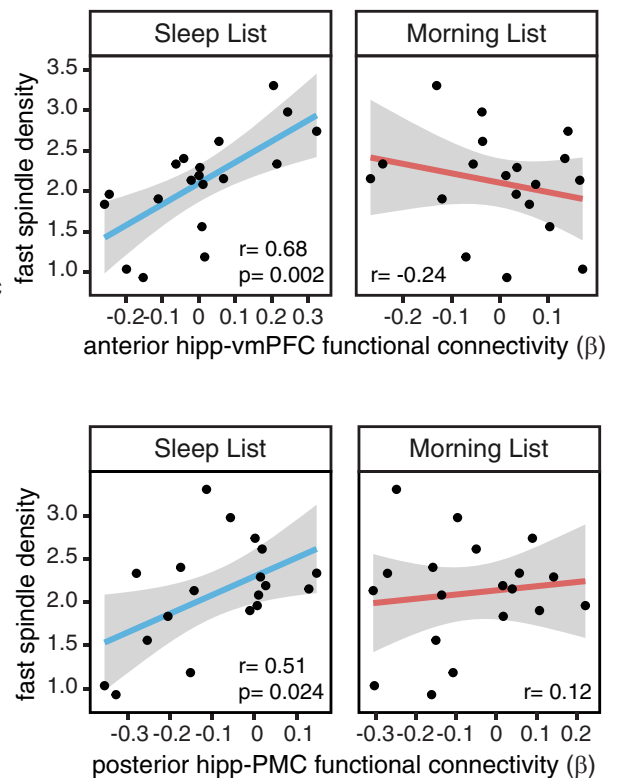

b

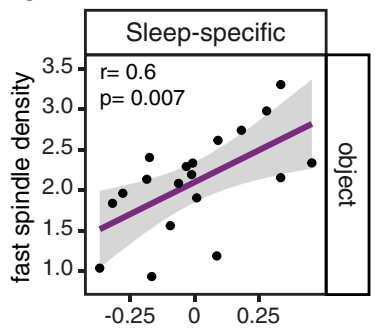

d

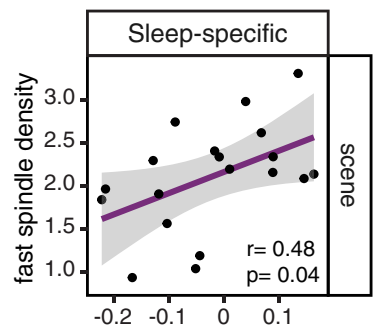

Figure 3. Hippocampal-neocortical functional connectivity correlates with fast spindle density for the Sleep List. $\boldsymbol{a}$, Greater fast spindle density during overnight sleep positively correlated with increased right anterior hippocampal-vmPFC functional connectivity only during restudy of the Sleep List (blue), but not the Morning List (red), object-word pairs. $\boldsymbol{b}$, In a control analysis, the sleep-specific functional connectivity (SL-ML) for object-word pairs still positively correlated with fast spindle density during overnight sleep. c, Greater fast spindle density positively correlated with right posterior hippocampal-PMC functional connectivity only for the Sleep List (blue), and not for Morning List (red), scene-word pairs. $\boldsymbol{d}$, Fast spindle density also positively correlated with the control, sleep-specific posterior hippocampal-PMC functional connectivity (SL-ML) for scene-word pairs. Black dots represent individual participants and gray ribbon indicates $95 \%$ confidence intervals.

fast spindle density $(r=0.51, p=0.02, \mathrm{BF}=4.83 ;$ Fig. $3 c)$. This relationship was not significant for the more recently learned Morning List pairs $(r=0.12, p=0.62, \mathrm{BF}=0.45)$, and these correlations again were significantly different from each other $(t=2.52, p=0.02$; Fig. $3 c)$. Additionally, there was a significant positive correlation between spindle density and the sleepspecific right posterior hippocampal-PMC functional connectivity measure $(r=0.48, p=0.04$; Fig. $3 d)$. There was no overall difference between the Sleep List and Morning List connectivity $\left(t_{(18)}=0.33, p=0.75\right)$.

Right posterior hippocampal-PMC functional connectivity during the object-word pair restudy did not significantly correlate with fast spindles for either list (SL: $r=0.1, p=0.69, \mathrm{BF}=$ 0.52 ; ML: $r=-0.06, p=0.82, \mathrm{BF}=0.49)$. A direct comparison of the Sleep List scene-word and object-word pair spindle-connectivity correlations was marginally different $(t=1.85, p=$ 0.08 ). There was also not a significant correlation between left posterior hippocampal functional connectivity with PMC and fast spindle density for the Sleep List scene-word pairs $(r=0.36$, $p=0.13)$, though the correlation was significant for the Morning List scene-word pairs $(r=0.51, p=0.03)$; however, these correlations were not significantly different $(t=0.89, p=0.39)$, and the correlation with the sleep-specific measure was not significant $(r=-0.26, p=0.28)$. Functional connectivity between right posterior hippocampus and right parahippocampal cortex marginally correlated with fast spindle density for the Sleep List scene-word pairs $(r=0.45, p=0.053)$, and not the Morning List $(r=-0.04, p=0.89)$, but connectivity between posterior hippocampus and vmPFC or PrC did not significantly correlate with fast spindle density.

Together, these results demonstrate that functional connectivity in both the anterior and posterior hippocampal-neocortical networks is related to fast spindle activity during the prior night of sleep. Critically, the hippocampal networks sensitive to fast spindles seemed to differ based on the content of the memories: fast spindles correlated with increased right anterior hippocampal-vmPFC connectivity when restudying the Sleep List object-word pairs, but with the right posterior hippocampalPMC functional network when restudying the Sleep List sceneword pairs. Importantly, these effects cannot be driven by the on-screen category presentation during the scanning session because the same effects were not evident for the Morning List object-word or scene-word pairs. This suggests that the networks supporting sleep-consolidated information are at least somewhat sensitive to the visual category of the stimulus. As such, we proceeded to focus on the category relevant to each network of interest in subsequent analyses.

\section{Inter-item pattern similarity}

Thus far, we have shown that fast spindle density during overnight sleep is associated with increased hippocampal-cortical network connectivity measured during the restudy of information learned before, but not after, the sleep period. We next wanted to test the hypothesis that sleep spindles play a role in the integrative, gist-extraction process, resulting in more overlapping neural representations for memories learned before sleep. We hypothesized that this consolidation-related gist extraction might be supported by the vmPFC, which has been implicated in the emergence and maintenance of schemas (Tse et al., 2007; van Kesteren et al., 2010, 2013b; Preston and Eichenbaum, 2013; Schlichting and Preston, 2015; Spalding et al., 2018), as well as the retrieval of remote, compared with recent, memories more broadly (Takashima et al., 2006, 2007, 2009; Gais et al., 2007; Sterpenich et al., 2007, 2009).

We used a multivariate pattern analysis to measure neural similarity between representations of individual memories from the same Encoding List (e.g., Sleep List) by extracting the BOLD activity pattern for each word-image pair (each trial during the 


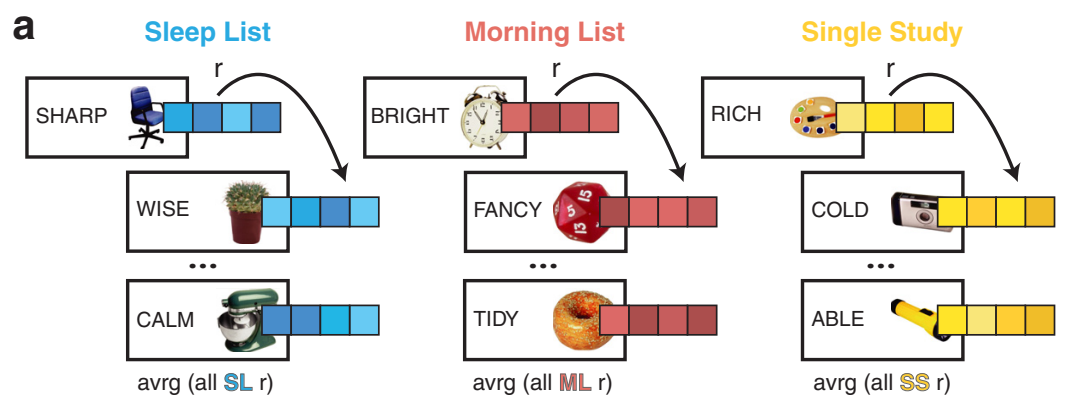

b

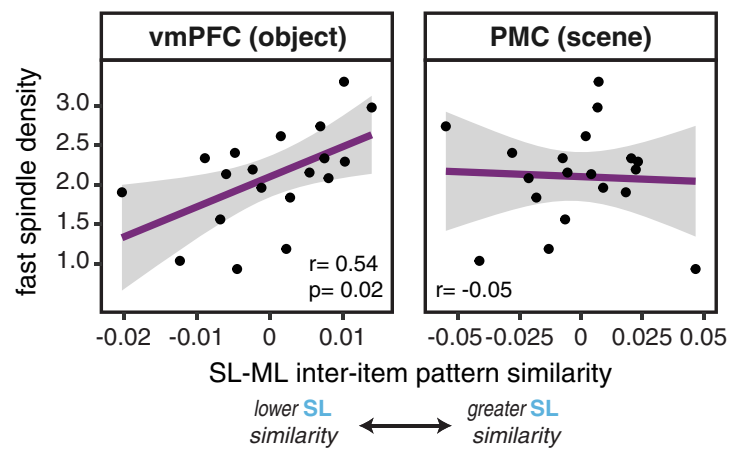

Figure 4. Inter-item pattern similarity. $\boldsymbol{a}$, To compute the inter-item pattern analysis, patterns of activation across voxels within ROls were extracted for each trial and sorted by the Encoding List it was first learned during. All trials within a list were correlated, across runs, and averaged, resulting in a similarity metric within each list. $\boldsymbol{b}$, Fast spindle density correlated with inter-item pattern similarity in vmPFC for object-word pairs (left: $r=0.54, p=0.017$ ) but not for scene-word pairs in PMC (right: $r=-0.05)$. For all plots, black dots represent individual participants and gray ribbon indicates $95 \%$ confidence intervals.

scan) and computing its similarity with all other trials encoded as part of the same list and within the same category (Fig. $4 a$; see Materials and Methods). We refer to this as an inter-item pattern similarity score.

We first examined whether there were differences in interitem pattern similarity between the Encoding Lists, separately for the object-word and scene-word pairs. In vmPFC, there was no main effect of Encoding List $\left(F_{(2,36)}=0.996, p=0.38\right)$, or list $\times$ category interaction effect $\left(F_{(2,36)}=0.41, p=0.67\right)$. However, we found significantly lower inter-item pattern similarity among object-word pairs compared with scene-word pairs (category main effect: $\left.F_{(1,18)}=5.67, p=0.03\right)$.

As with the functional connectivity analyses, we investigated whether fast spindle density during overnight sleep was related to the inter-item pattern similarity measured during the Sleep List versus Morning List restudy trials. We found that, for objectword pairs, the sleep-specific inter-item pattern similarity in vmPFC significantly correlated with overnight fast spindle density $(r=0.54, p=0.02, \mathrm{BF}=4.44$; Fig. $4 b)$. In other words, greater fast spindle density was associated with greater pattern similarity, or representational overlap, among the Sleep List object-word pairs, relative to those studied in the morning. For scene-word pairs, this sleep-specific similarity-spindle relationship was not significant $(r=0.11, p=0.67, \mathrm{BF}=0.52)$, though these two correlations were not significantly different from each other $(t=1.32, p=0.21)$.

Next we examined inter-item pattern similarity in PMC. We again found that similarity was greater across scene-word compared with object-word pairs (category main effect: $F_{(1,18)}=$ $30.79, p<0.0001)$, as in vmPFC. There was not a significant main effect of list $\left(F_{(2,36)}=1.92, p=0.16\right)$, or interaction effect $\left(F_{(2,36)}\right.$ $=0.4, p=0.67)$. Based on the above functional connectivity results involving PMC, we first examined the spindle-similarity relationship specifically for the sceneword pairs. However, this correlation was not significant $(r=-0.05, p=0.84, \mathrm{BF}=$ 0.49; Fig. 4b). Fast spindle density also did not correlate with sleep-specific interitem pattern similarity in PMC for objectword pairs $(r=0.29, p=0.23, \mathrm{BF}=0.84)$. A direct test showed a marginal difference between the spindle-similarity correlations for the vmPFC (object-word pairs) and PMC (scene-word pairs; $t=1.95, p=$ 0.07 ). Thus, it seems that fast spindles may particularly affect representational similarity in $\mathrm{vmPFC}$

Additionally, we examined the interitem pattern similarity in $\mathrm{PhC}$ and $\mathrm{PrC}$; however, we found no significant relationships between fast spindle density and sleep-specific pattern similarity in these regions. Overall, for both right and left PrC inter-item pattern similarity differed by category, with object-word pairs more similar than scene-word pairs (main effect of category: right $\operatorname{PrC}, F_{(1,18)}=8.19$, $p=0.01$; left $\operatorname{PrC}, F_{(1,18)}=18.93, p=$ $0.0004)$, but was not modulated by the Encoding List (right: $F_{(2,36)}=1.85, p=$ 0.17 ; left: $\left.F_{(2,36)}=1.54, p=0.23\right)$. In contrast, for right $\mathrm{PhC}$, inter-item pattern similarity was greater for scene-word pairs compared with object-word pairs (category main effect: $F_{(1,18)}=5.02, p=0.04$ ) and for similarity differed by Encoding List for both right and left $\mathrm{PhC}$ (main effect of list: right, $F_{(2,36)}=$ $6.06, p=0.005$; left, $\left.F_{(2,36)}=4.1, p=0.025\right)$.

\section{Relationship between functional connectivity and inter-item pattern similarity}

Thus far, our results indicate that, when reactivating information originally learned before sleep, fast spindles during overnight sleep are correlated with (1) greater anterior hippocampalvmPFC functional connectivity for object-word pairs, (2) greater posterior hippocampal-PMC functional connectivity for sceneword pairs, and (3) greater pattern similarity in vmPFC for object-word pairs. As theories suggest that memory representations are reorganized via extended hippocampal-cortical communication, we expected that our two neural measures showing effects for the Sleep List, hippocampal-cortical functional connectivity, and the degree of cortical representational overlap would be related. Since we were particularly interested in whether this relationship was dependent on later memory success, we conducted this analysis separately for later remembered and forgotten pairs (see Materials and Methods).

For later remembered object-word pairs, the sleep-specific right anterior hippocampal-vmPFC functional connectivity did indeed positively correlate with the inter-item pattern similarity in vmPFC for those pairs $(r=0.62, p=0.005, \mathrm{BF}=10.61$; Fig. 5$)$. In other words, for Sleep List memories, greater functional connectivity was associated with more overlapping representations in vmPFC. This relationship was specific to trials that were later remembered; there was not a significant correlation between the measures for forgotten pairs $(r=-0.09, p=0.70, \mathrm{BF}=0.51$; Fig. $5)$. The sleep-specific connectivity-similarity correlations for re- 


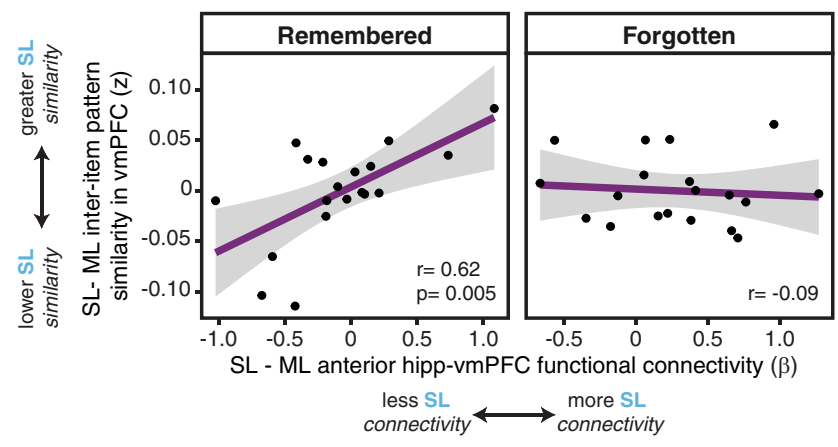

Figure 5. Relationship between right anterior hippocampal-vmPFC functional connectivity and inter-item pattern similarity in vmPFC. For remembered object-word pairs (left), sleepspecific functional connectivity positively correlates with sleep-specific inter-item pattern similarity $(r=0.62, p=0.005)$, such that greater connectivity for the Sleep List, relative to the Morning List, was related to greater similarity across these pairs in vmPFC. For object-word pairs that were subsequently forgotten (right), anterior hippocampal-vmPFC functional connectivity did not correlate with the representational similarity $(r=-0.09)$. These correlations are significantly different from one another $(z=2.17, p=0.03)$. For all plots, black dots represent individual participants and gray ribbon indicates $95 \%$ confidence intervals.

membered and forgotten pairs were significantly different from each other $(z=2.17, p=0.03)$.

There was no such relationship, however, for the scene-word pairs $(r=-0.35, p=0.14)$, which is consistent with the emerging pattern of results. Together, these results suggest that for objectbased memories given the opportunity for sleep-dependent consolidation, the extent of functional connectivity between the anterior hippocampus and vmPFC is related to the overlap in the representations in vmPFC for those memories.

We next examined whether posterior hippocampal-PMC functional connectivity was related to the representational structure in PMC. We focused the analysis on scene-word pairs, as fast spindle density was related to the functional connectivity for this category. Unlike the results reported above in vmPFC, there was no significant correlation between the sleep-specific functional connectivity and inter-item pattern similarity in PMC $(r=0.09$, $p=0.73)$. According to a direct statistical test, the correlations for vmPFC and PMC were significantly different $(z=1.93, p=$ 0.05 ), suggesting a dissociation between these two midline neocortical brain regions.

\section{Mediation analysis: fast spindles, functional connectivity, and} representational overlap

Together, the results presented so far suggest that the coactivation between the anterior hippocampus and vmPFC may be related to the extent of local transformation of the Sleep List object-word memory traces in vmPFC. Spindles are theorized to help facilitate the information transfer through this hippocampal-cortical dialogue (Siapas and Wilson, 1998; Sirota et al., 2003; Steriade, 2006; Rasch and Born, 2013; Staresina et al., 2015), and consolidation theories posit that, through the cross-regional communication, the cortex builds up an integrated memory trace (McClelland et al., 1995), which suggests that the mechanism of spindles for affecting the representational structure of cortical traces may be indirect, depending on the strength of the functional connectivity.

Using a mediation analysis, we formally tested whether the relationship between fast spindle density and sleep-specific interitem pattern similarity for remembered object-word pairs in vmPFC is mediated by the sleep-specific anterior hippocampalvmPFC functional connectivity for those same trials (Fig. 6; see Materials and Methods). Indeed, we found that the direct rela-

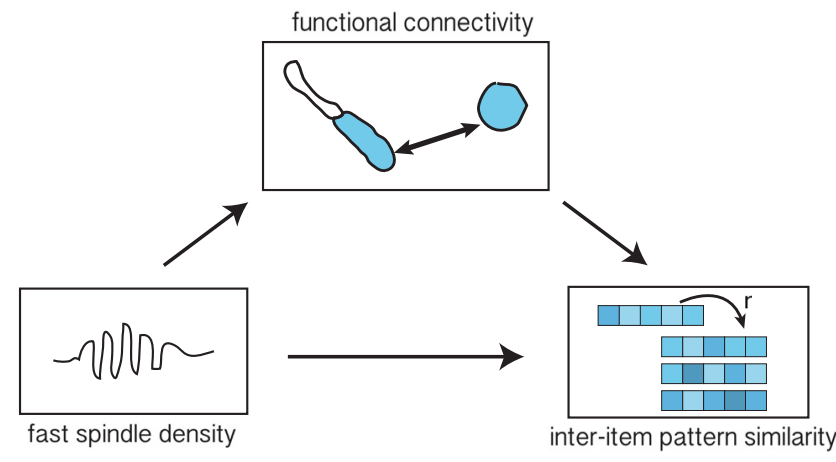

Figure 6. Schematic illustration of mediation analysis. A significant mediation effect demonstrated that fast spindle density during overnight sleep is related to greater right anterior hippocampal-vmPFC functional connectivity for the sleep-specific remembered object-word pairs and, through this relationship, is indirectly associated with greater representational overlap in vmPFC among these pairs.

tionship between overnight fast spindles and next-day vmPFC representational overlap was attenuated by the addition of the anterior hippocampal-vmPFC functional connectivity in the model, resulting in a significant mediation effect $(\mathrm{ACME}=0.02$, $p=0.039$ ), as assessed using the bootstrapping method (see Materials and Methods). This analysis suggests that fast spindles exert their effects on memory representations in vmPFC through their direct modulation of anterior hippocampal-vmPFC functional connectivity.

\section{Discussion}

The current study combined overnight polysomnography, nextday fMRI, and behavioral measures of associative memory, to probe whether, and how, fast sleep spindles promote lasting changes in neural memory traces. We tested three specific questions, asking whether fast spindles during overnight sleep after learning were associated with the following: (1) strengthening of hippocampal-cortical functional networks measured the next day, (2) changes in the representational structure of presleep memories in cortical regions, and, critically, (3) the mechanistic relationship between these measures. Fast spindles during overnight sleep were related to the next-day neural representation of information learned before, and importantly, not after, the sleep period. In particular, fast spindle density positively correlated with functional connectivity in both anterior and posterior hippocampal-neocortical networks. Fast spindle density also correlated with greater overlap among the multivariate pattern representations in vmPFC for the object-word pairs learned before sleep. Further, the representational overlap in vmPFC positively correlated with the extent of anterior hippocampal-vmPFC functional connectivity for the successfully remembered objectword pairs, and a significant mediation analysis demonstrated that fast spindles promote this representational overlap in vmPFC through the enhancement of anterior hippocampalvmPFC functional connectivity. Together, these results provide new evidence suggesting that fast spindles during overnight sleep may alter the neural representation of memory traces, distributing memories between hippocampal-cortical networks, and through this cross-regional communication, promoting the restructuring of memory traces within the vmPFC.

Sleep spindles are proposed to play an important role in systems memory consolidation. Considerable evidence has linked spindles during postlearning sleep to postsleep memory retention benefits (Gais et al., 2002; Schabus et al., 2004; Clemens et al., 
2006; Mednick et al., 2013; Rasch and Born, 2013; Hennies et al., 2016). Current theories suggest that spindles, through the temporal coupling with sharp-wave ripples and slow oscillations, facilitate the coordination of reactivated memories from hippocampus to cortex (Siapas and Wilson, 1998; Sirota et al., 2003; Steriade, 2006; Clemens et al., 2007; Mölle and Born, 2011; Rasch and Born, 2013; Staresina et al., 2015). Evidence for this theory has generally come from studying brain dynamics during sleep; fast spindle activity has been linked with overall activation in hippocampus and cortex (Schabus et al., 2007; Bergmann et al., 2012), functional connectivity measures (Andrade et al., 2011), and with memory reactivation during sleep (Schönauer et al., 2017; Antony et al., 2018; Cairney et al., 2018). The current study adds to this growing body of work by showing that spindles not only have transitory effects on the brain during sleep, but also contribute to lasting neural changes associated with the distribution and representation of those memories. Our results show that postlearning fast spindle density is associated with next-day enhancements in hippocampal-cortical functional connectivity specifically for the memories learned before sleep, and through this functional pathway, spindles also promoted the local reorganization of multivariate representations in vmPFC.

As reported here, the representational organization in vmPFC, but not PMC, was sensitive to fast spindle density, despite significant spindle-connectivity relationships for these regions with anterior and posterior hippocampus, respectively. One possible explanation is that vmPFC representations are particularly reliant on sleep-dependent consolidation. Prior work suggests that consolidation modulates retrieval-related activity in vmPFC (Takashima et al., 2006, 2007, 2009; Gais et al., 2007; Sterpenich et al., 2007, 2009; Sekeres et al., 2018b). Further, processes within vmPFC are thought to promote generalization across memories, including the emergence of schemas and the integration of memories with overlapping content (van Kesteren et al., 2010, 2013a; Preston and Eichenbaum, 2013; Richards et al., 2014; Schlichting and Preston, 2015; Tompary and Davachi, 2017; Spalding et al., 2018), which aligns with theories suggesting neocortical regions come to represent the gist, or central tendencies, of new memories with consolidation (McClelland et al., 1995; Lewis and Durrant, 2011; Winocur and Moscovitch, 2011; Sekeres et al., 2018a). Here, fast spindle density during overnight sleep correlated with greater multivariate pattern similarity in representations for object-word pairs learned before sleep, which may be indicative of generalization across these memories. Thus, spindles may act as a physiological mechanism, via functional networks, for the neural integration of vmPFC traces. This may be adaptive for memory retention, as only later remembered pairs showed a significant correlation between anterior hippocampal-vmPFC functional connectivity and representational overlap. However, since our memory measure was a fairly general source memory test, future work will be needed to directly test the behavioral consequences of such neural representational changes.

In contrast to vmPFC, mnemonic representations in PMC may be more important in maintaining episodic detail rather than representing similarities across memories. Prior work has implicated PMC in episodic detail reinstatement during shortand long-term retrieval (Bird et al., 2015; Chen et al., 2017), with little evidence for integration across memories sharing overlapping content (Tompary and Davachi, 2017). It is thus possible that fast spindles could promote the stabilization of individual episodic memories in PMC, but future work will be needed to address this specificity.
The results presented here suggest that sleep-dependent consolidation effects may also be localized to specific networks depending on the content of the presleep learning experience. A prior study measuring simultaneous EEG-fMRI during sleep reported that fast spindle amplitude modulated univariate activation selectively in brain regions activated during the preceding encoding task (Bergmann et al., 2012). Likewise, reports have shown experience-dependent changes in postencoding hippocampal functional connectivity with cortical regions previously engaged during encoding (Tambini et al., 2010; van Kesteren et al., 2010; Vilberg and Davachi, 2013; Schlichting and Preston, 2014; Murty et al., 2017; Collins and Dickerson, 2019). Here, in line with the proposed dichotomy in anterior and posterior hippocampal networks and functional specialization (Davachi, 2006; Libby et al., 2012; Ranganath and Ritchey, 2012; Poppenk et al., 2013; Robin and Moscovitch, 2017), we found that fast spindle density correlated with next-day anterior hippocampal-vmPFC and posterior hippocampal-PMC functional connectivity for the object-word and scene-word pairs, respectively. Importantly, these effects cannot be explained by perceptual differences between the categories alone; if the fast spindle-connectivity correlations only reflected processing of the stimuli on screen, the pairs learned in the morning should show the same results, yet no spindle-connectivity correlations were significant for the Morning List. This is particularly important as neural responses were measured during restudy, a design adopted to allow equal opportunity to reactivate all previously studied pairs without engaging in an explicit memory search, thus avoiding potential recency confounds when retrieving the Morning List over the Sleep List. Further, differences in memory retention cannot explain the specificity of these effects on presleep information, as associative memory performance for the Sleep List and Morning List was matched on both memory tests.

There are some limitations to the current experiment that warrant further consideration. While our data suggest that sleep may promote more similar representational patterns in vmPFC, other research has shown that years-old autobiographical memories become more distinctive and amenable to decoding in vmPFC (Bonnici et al., 2012; Bonnici and Maguire, 2018). These results are not in direct conflict with our findings, as the extent of representational overlap or distinctiveness may depend on many factors, including similarity in the content of the memories (Tompary and Davachi, 2017) and the learning schedule (Ezzyat et al., 2018). In autobiographical memory studies, the procedure of choosing personal memories that are remembered years later could also result in the selection of memories that are distinctive, and hence represented in different vmPFC networks. Future work should examine how the content and context of memories alter their representational overlap in vmPFC, providing a deeper understanding of how experiences are integrated or kept distinctive in this, and other, cortical regions.

Additionally, the design of this experiment necessitated unequal encoding-restudy delay periods for the Sleep List and Morning List; to examine how spindles contribute to next-day organization of memories in the scanner, we compared memories learned before the sleep period with those that did not have an opportunity for sleep-dependent consolidation. While our results suggest that fast spindles selectively influence representations for the content encountered prior to sleep, more work is needed to directly compare the effects of an equal wake delay. For example, the overall magnitude of functional connectivity did not generally differ between the Sleep List and the Morning List, potentially due to consolidation-related enhancements that also 
occurred during the wake delay. Thus, more work is necessary to tease apart the contributions and mechanisms of sleep versus wake in consolidation.

For decades, research has linked memory improvements with sleep, with recent research focused on understanding the physiological events during sleep, such as spindles, that contribute to these effects. We conducted a multimodal, multiday study to ascertain whether, and how, fast spindles are related to neural markers of consolidation that endure after sleep. In demonstrating that fast spindle-related reorganization involves lasting enhancements in hippocampal-cortical functional network connectivity and the restructuring of vmPFC memory representations, this report provides critical evidence that sleep does more than just stabilize memories, and indeed supports enduring changes in the organization of memories both within, and across, regions of the brain that may support the retention of memories over time.

\section{References}

Alvarez P, Squire LR (1994) Memory consolidation and the medial temporal lobe: a simple network model. Proc Natl Acad Sci U S A 91:7041-7045.

Andrade KC, Spoormaker VI, Dresler M, Wehrle R, Holsboer F, Sämann PG, Czisch M (2011) Sleep spindles and hippocampal functional connectivity in human NREM sleep. J Neurosci 31:10331-10339.

Antony JW, Piloto L, Wang M, Pacheco P, Norman KA, Paller KA (2018) Sleep spindle refractoriness segregates periods of memory reactivation. Curr Biol 28:1736-1743.e4.

Baran B, Mantua J, Spencer RM (2016) Age-related changes in the sleepdependent reorganization of declarative memories. J Cogn Neurosci 28: 792-802.

Bergmann TO, Mölle M, Diedrichs J, Born J, Siebner HR (2012) Sleep spindle-related reactivation of category-specific cortical regions after learning face-scene associations. Neuroimage 59:2733-2742.

Berry RB, Budhiraja R, Gottlieb DJ, Gozal D, Iber C, Kapur VK, Marcus CL, Mehra R, Parthasarathy S, Quan SF, Redline S, Strohl KP, Davidson Ward SL, Tangredi MM (2012) Rules for scoring respiratory events in sleep: update of the 2007 AASM manual for the scoring of sleep and associated events. J Clin Sleep Med 8:597-619.

Bird CM, Keidel JL, Ing LP, Horner AJ, Burgess N (2015) Consolidation of complex events via reinstatement in posterior cingulate cortex. J Neurosci 35:14426-14434.

Bonnici HM, Maguire EA (2018) Two years later-Revisiting autobiographical memory representations in vmPFC and hippocampus. Neuropsychologia 110:159-169.

Bonnici HM, Chadwick MJ, Lutti A, Hassabis D, Weiskopf N, Maguire EA (2012) Detecting representations of recent and remote autobiographical memories in vmPFC and hippocampus. J Neurosci 32:16982-16991.

Brady TF, Konkle T, Alvarez GA, Oliva A (2008) Visual long-term memory has a massive storage capacity for object details. Proc Natl Acad Sci U S A 105:14325-14329.

Cairney SA, Guttesen AÁV, El Marj N, Staresina BP (2018) Memory consolidation is linked to spindle-mediated information processing during sleep. Curr Biol 28:948-954.e4.

Chen J, Leong YC, Honey CJ, Yong CH, Norman KA, Hasson U (2017) Shared memories reveal shared structure in neural activity across individuals. Nat Neurosci 20:115-125.

Clemens Z, Fabó D, Halász P (2005) Overnight verbal memory retention correlates with the number of sleep spindles. Neuroscience 132:529-535.

Clemens Z, Fabó D, Halász P (2006) Twenty-four hours retention of visuospatial memory correlates with the number of parietal sleep spindles. Neurosci Lett 403:52-56.

Clemens Z, Mölle M, Eross L, Barsi P, Halász P, Born J (2007) Temporal coupling of parahippocampal ripples, sleep spindles and slow oscillations in humans. Brain 130:2868-2878.

Clemens Z, Mölle M, Eross L, Jakus R, Rásonyi G, Halász P, Born J (2011) Fine-tuned coupling between human parahippocampal ripples and sleep spindles. Eur J Neurosci 33:511-520.

Collins JA, Dickerson BC (2019) Functional connectivity in categoryselective brain networks after encoding predicts subsequent memory. Hippocampus 29:440-450.
Davachi L (2006) Item, context and relational episodic encoding in humans. Curr Opin Neurobiol 16:693-700.

Diekelmann S, Born J (2010) The memory function of sleep. Nat Rev Neurosci 11:114-126.

Eichenbaum H (2017) Prefrontal-hippocampal interactions in episodic memory. Nat Rev Neurosci 18:547-558.

Ezzyat Y, Inhoff MC, Davachi L (2018) Differentiation of human medial prefrontal cortex activity underlies long-term resistance to forgetting in memory. J Neurosci 38:10244-10254.

Gais S, Mölle M, Helms K, Born J (2002) Learning-dependent increases in sleep spindle density. J Neurosci 22:6830-6834.

Gais S, Albouy G, Boly M, Dang-Vu TT, Darsaud A, Desseilles M, Rauchs G, Schabus M, Sterpenich V, Vandewalle G, Maquet P, Peigneux P (2007) Sleep transforms the cerebral trace of declarative memories. Proc Natl Acad Sci U S A 104:18778-18783.

Hennies N, Lambon Ralph MA, Kempkes M, Cousins JN, Lewis PA (2016) Sleep spindle density predicts the effect of prior knowledge on memory consolidation. J Neurosci 36:3799-3810.

Hoscheidt SM, Nadel L, Payne J, Ryan L (2010) Hippocampal activation during retrieval of spatial context from episodic and semantic memory. Behav Brain Res 212:121-132.

Klinzing JG, Mölle M, Weber F, Supp G, Hipp JF, Engel AK, Born J (2016) Spindle activity phase-locked to sleep slow oscillations. Neuroimage 134: 607-616.

LaRocque KF, Smith ME, Carr VA, Witthoft N, Grill-Spector K, Wagner AD (2013) Global similarity and pattern separation in the human medial temporal lobe predict subsequent memory. J Neurosci 33:5466-5474.

Lewis PA, Durrant SJ (2011) Overlapping memory replay during sleep builds cognitive schemata. Trends Cogn Sci 15:343-351.

Libby LA, Ekstrom AD, Ragland JD, Ranganath C (2012) Differential connectivity of perirhinal and parahippocampal cortices within human hippocampal subregions revealed by high-resolution functional imaging. J Neurosci 32:6550-6560.

McClelland JL, McNaughton BL, O'Reilly RC (1995) Why there are complementary learning systems in the hippocampus and neo-cortex: insights from the successes and failures of connectionists models of learning and memory. Psychol Rev 102:419-457.

Mednick SC, McDevitt EA, Walsh JK, Wamsley E, Paulus M, Kanady JC, Drummond SP (2013) The critical role of sleep spindles in hippocampaldependent memory: a pharmacology study. J Neurosci 33:4494-4504.

Mölle M, Born J (2011) Slow oscillations orchestrating fast oscillations and memory consolidation. Prog Brain Res 193:93-110.

Mölle M, Marshall L, Gais S, Born J (2002) Grouping of spindle activity during slow oscillations in human non-rapid eye movement sleep. J Neurosci 22:10941-10947.

Mölle M, Bergmann TO, Marshall L, Born J (2011) Fast and slow spindles during the sleep slow oscillation: disparate coalescence and engagement in memory processing. Sleep 34:1411-1421.

Mumford JA, Davis T, Poldrack RA (2014) The impact of study design on pattern estimation for single-trial multivariate pattern analysis. Neuroimage 103:130-138.

Murty VP, Tompary A, Adcock RA, Davachi L (2017) Selectivity in postencoding connectivity with high-level visual cortex is associated with reward-motivated memory. J Neurosci 37:537-545.

Nadel L, Hoscheidt S, Ryan LR (2013) Spatial cognition and the hippocampus: the anterior-posterior axis. J Cogn Neurosci 25:22-28.

Oliva A, Torralba A (2001) Modeling the shape of the scene: a holistic representation of the spatial envelope. Int J Comput Vis 42:145-175.

Poppenk J, Evensmoen HR, Moscovitch M, Nadel L (2013) Long-axis specialization of the human hippocampus. Trends Cogn Sci 17:230-240.

Preston AR, Eichenbaum H (2013) Interplay of hippocampus and prefrontal cortex in memory. Curr Biol 23:R764-R773.

Ranganath C, Ritchey M (2012) Two cortical systems for memory-guided behaviour. Nat Rev Neurosci 13:713-726.

Rasch B, Born J (2013) About sleep's role in memory. Physiol Rev 93: 681-766.

Richards BA, Xia F, Santoro A, Husse J, Woodin MA, Josselyn SA, Frankland PW (2014) Patterns across multiple memories are identified over time. Nat Neurosci 17:981-986.

Robin J, Moscovitch M (2017) Details, gist and schema: hippocampalneocortical interactions underlying recent and remote episodic and spatial memory. Curr Opin Behav Sci 17:114-123. 
Schabus M, Gruber G, Parapatics S, Sauter C, Klösch G, Anderer P, Klimesch W, Saletu B, Zeitlhofer J (2004) Sleep spindles and their significance for declarative memory consolidation. Sleep 27:1479-1485.

Schabus M, Dang-Vu TT, Albouy G, Balteau E, Boly M, Carrier J, Darsaud A, Degueldre C, Desseilles M, Gais S, Phillips C, Rauchs G, Schnakers C, Sterpenich V, Vandewalle G, Luxen A, Maquet P (2007) Hemodynamic cerebral correlates of sleep spindles during human non-rapid eye movement sleep. Proc Natl Acad Sci U S A 104:13164-13169.

Schlichting ML, Preston AR (2014) Memory reactivation during rest supports upcoming learning of related content. Proc Natl Acad Sci U S A 111:15845-15850.

Schlichting ML, Preston AR (2015) Memory integration: neural mechanisms and implications for behavior. Curr Opin Behav Sci 1:1-8.

Schönauer M, Alizadeh S, Jamalabadi H, Abraham A, Pawlizki A, Gais S (2017) Decoding material-specific memory reprocessing during sleep in humans. Nat Commun 8:15404

Sekeres MJ, Winocur G, Moscovitch M (2018a) The hippocampus and related neocortical structures in memory transformation. Neurosci Lett 680:39-53.

Sekeres MJ, Winocur G, Moscovitch M, Anderson JAE, Pishdadian S, Martin Wojtowicz J, St-Laurent M, McAndrews MP, Grady CL (2018b) Changes in patterns of neural activity underlie a time-dependent transformation of memory in rats and humans. Hippocampus 28:745-764.

Shirer WR, Ryali S, Rykhlevskaia E, Menon V, Greicius MD (2012) Decoding subject-driven cognitive states with whole-brain connectivity patterns. Cereb Cortex 22:158-165.

Shrout PE, Bolger N (2002) Mediation in experimental and nonexperimental studies: new procedures and recommendations. Psychol Methods $7: 422-445$.

Siapas AG, Wilson MA (1998) Coordinated interactions between hippocampal ripples and cortical spindles during slow-wave sleep. Neuron 21:1123-1128.

Sirota A, Csicsvari J, Buhl D, Buzsáki G (2003) Communication between neocortex and hippocampus during sleep in rodents. Proc Natl Acad Sci U S A 100:2065-2069.

Spalding KN, Schlichting ML, Zeithamova D, Preston AR, Tranel D, Duff MC, Warren DE (2018) Ventromedial prefrontal cortex is necessary for normal associative inference and memory integration. J Neurosci 38:3767-3775.

Staresina BP, Bergmann TO, Bonnefond M, van der Meij R, Jensen O, Deuker L, Elger CE, Axmacher N, Fell J (2015) Hierarchical nesting of slow oscillations, spindles and ripples in the human hippocampus during sleep. Nat Neurosci 18:1679-1686.

Stark CE, Squire LR (2001) When zero is not zero: the problem of ambiguous baseline conditions in fMRI. Proc Natl Acad Sci US A 98:1276012766.

Steriade M (2006) Grouping of brain rhythms in corticothalamic systems. Neuroscience 137:1087-1106.

Sterpenich V, Albouy G, Boly M, Vandewalle G, Darsaud A, Balteau E,
Dang-Vu TT, Desseilles M, D’Argembeau A, Gais S, Rauchs G, Schabus M, Degueldre C, Luxen A, Collette F, Maquet P (2007) Sleep-related hippocampo-cortical interplay during emotional memory recollection. PLoS Biol 5:e282.

Sterpenich V, Albouy G, Darsaud A, Schmidt C, Vandewalle G, Dang Vu TT, Desseilles M, Phillips C, Degueldre C, Balteau E, Collette F, Luxen A, Maquet P (2009) Sleep promotes the neural reorganization of remote emotional memory. J Neurosci 29:5143-5152.

Takashima A, Petersson KM, Rutters F, Tendolkar I, Jensen O, Zwarts MJ, McNaughton BL, Fernández G (2006) Declarative memory consolidation in humans: a prospective functional magnetic resonance imaging study. Proc Natl Acad Sci U S A 103:756-761.

Takashima A, Nieuwenhuis IL, Rijpkema M, Petersson KM, Jensen O, Fernández G (2007) Memory trace stabilization leads to large-scale changes in the retrieval network: a functional MRI study on associative memory. Learn Mem 14:472-479.

Takashima A, Nieuwenhuis IL, Jensen O, Talamini LM, Rijpkema M, Fernández G (2009) Shift from hippocampal to neocortical centered retrieval network with consolidation. J Neurosci 29:10087-10093.

Tambini A, Ketz N, Davachi L (2010) Enhanced brain correlations during rest are related to memory for recent experiences. Neuron 65:280-290.

Tompary A, Davachi L (2017) Consolidation promotes the emergence of representational overlap in the hippocampus and medial prefrontal cortex. Neuron 96:228-241.e5.

Tse D, Langston RF, Kakeyama M, Bethus I, Spooner PA, Wood ER, Witter MP, Morris RG (2007) Schemas and memory consolidation. Science 316:76-82.

van Kesteren MT, Fernández G, Norris DG, Hermans EJ (2010) Persistent schema-dependent hippocampal-neocortical connectivity during memory encoding and postencoding rest in humans. Proc Natl Acad Sci U S A 107:7550-7555.

van Kesteren MT, Beul SF, Takashima A, Henson RN, Ruiter DJ, Fernández G (2013a) Differential roles for medial prefrontal and medial temporal cortices in schema-dependent encoding: from congruent to incongruent. Neuropsychologia 51:2352-2359.

van Kesteren MT, Rijpkema M, Ruiter DJ, Fernández G (2013b) Consolidation differentially modulates schema effects on memory for items and associations. PLoS One 8:e56155.

Vilberg KL, Davachi L (2013) Perirhinal-hippocampal connectivity during reactivation is a marker for object-based memory consolidation. Neuron 79:1232-1242.

Wamsley EJ, Tucker MA, Shinn AK, Ono KE, McKinley SK, Ely AV, Goff DC, Stickgold R, Manoach DS (2012) Reduced sleep spindles and spindle coherence in schizophrenia: mechanisms of impaired memory consolidation? Biol Psychiatry 71:154-161.

Wilson MA, McNaughton BL (1994) Reactivation of hippocampal ensemble memories during sleep. Science 265:676-679.

Winocur G, Moscovitch M (2011) Memory transformation and systems consolidation. J Int Neuropsychol Soc 17:766-780. 